Case Report

\title{
Gastric Volvulus and Wandering Spleen: A Rare Surgical Emergency
}

\author{
Georgios Lianos, Konstantinos Vlachos, Nikolaos Papakonstantinou, Christos Katsios, \\ Georgios Baltogiannis, and Dimitrios Godevenos
}

Division of Surgery, University Hospital of Ioannina, St. Niarchou Avenue, 45110 Ioannina, Greece

Correspondence should be addressed to Georgios Lianos; georgiolianos@yahoo.gr

Received 9 December 2012; Accepted 11 January 2013

Academic Editors: J. Griniatsos, G. Rallis, M. Rangarajan, and A. Spinelli

Copyright (c) 2013 Georgios Lianos et al. This is an open access article distributed under the Creative Commons Attribution License, which permits unrestricted use, distribution, and reproduction in any medium, provided the original work is properly cited.

\begin{abstract}
Gastric volvulus is a rare but potentially life-threatening clinical entity due to possible gastric necrosis. A wandering spleen may also be associated with gastric volvulus. Patients presenting with the triad epigastralgia, vomiting followed by retching, and difficulty or inability to pass a nasogastric tube into the stomach are likely to have gastric volvulus. The operating surgeon should include this rare entity in the differential diagnosis when dealing with a patient with such a clinical profile. Herein, we present a case of gastric volvulus associated with a wandering spleen in a 28 -year-old Caucasian woman and we provide a brief review of the literature on this issue.
\end{abstract}

\section{Introduction}

Gastric volvulus is an extremely rare clinical entity first described by Berti in 1866 [1]. An abnormal rotation $\left(180^{\circ}\right)$ of one part of the stomach around another, potentially leading to obstruction of the gastric cavity is defined gastric volvulus. Volvulus may be organoaxial or mesenteroaxial, occuring around an axis made by two fixed points. When untreated, complete volvulus results in strangulation, which may lead to ischaemia, necrosis, and finally to gastric perforation. Interestingly, mortality rates can achieve levels of $30-50 \%$ $[2,3]$. Therefore, gastric volvulus is a true and life-threatening surgical emergency if not treated in time. Wandering spleen is also a rare entity characterized by the underdevelopment or complete absence of one or all of the ligaments that hold the spleen in its normal position. Moreover, gastric volvulus and wandering spleen share a common cause, the absence of intraperitoneal visceral ligaments $[4,5]$. We hereby present the rare case of a 28-year-old Caucasian woman with gastric volvulus associated with a wandering spleen. We share our experience in successful treatment of this unique case.

\section{Case Report}

A 28-year-old Caucasian female was admitted to the emergency department of our hospital complaining of severe abdominal pain, nausea, and multiple episodes of bilious vomiting followed by repeating nonproductive retching. Her medical history was unremarkable. Her vital signs showed only mild tachycardia. Upon physical examination the abdominal sounds were present and her abdomen was diffusely tender especially in the upper quadrant. No peptic ulcers or diaphragmatic hernias were included in her family history. Rectal examination showed an empty rectum. The laboratory tests were between normal ranges. There was a significant difficulty in passing a nasogastric tube which finally suctioned out a large amount of gastric fluid. The admission chest X-ray showed an enlarged stomach and an elevated left hemidiaphragm; so a gastrografin swallow was arranged. The latter showed a well-defined "bird beak" sign and a helical trend of the nasogastric tube (Figure 1). Gastroscopy was performed and revealed a large amount of gastric fluid in the stomach and mucosal ischemic lesions. Interestingly, the endoscope failed to intubate the pylorus. 


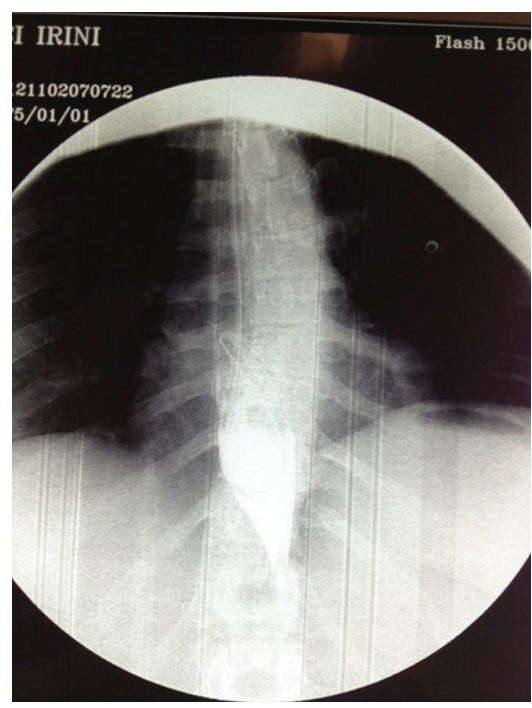

Figure 1: "Bird beak" sign and helical trend of the nasogastric tube after gastrografin swallow.

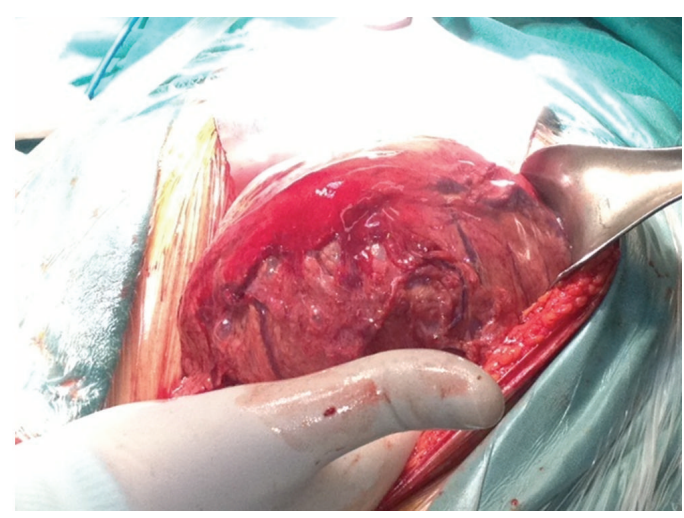

FIgURE 2: Twisted stomach (gastric volvulus).

An exploratory laparotomy was performed under general anesthesia the day after by midline incision. Intraoperative findings revealed a twisted distended stomach with ischemic lesions. A mesenteroaxial gastric volvulus was identified (Figures 2, 3, and 4 ). Interestingly, there was a lack of ligaments; so a wandering spleen was observed (Figure 5). The volvulus was untwisted and an approximation of the gastroesophageal junction and pylorus, predisposing to volvulus, was revealed (Figure 6). The stomach was reduced at its anatomic position, as well as the spleen, and an anterior gastropexy was carried out by fixing the greater curvature of the stomach to the anterior abdominal wall (Figure 7). The postoperative period was uneventful and the patient was discharged 10 days later. Two months after the operation, the patient remained asymptomatic.

\section{Discussion}

Gastric volvulus is a rare entity with difficult diagnosis. The incidence is unknown [6]. An abnormal rotation of one part



FIgURE 3: The untwisted distended stomach with ischemic mucosal lesions.

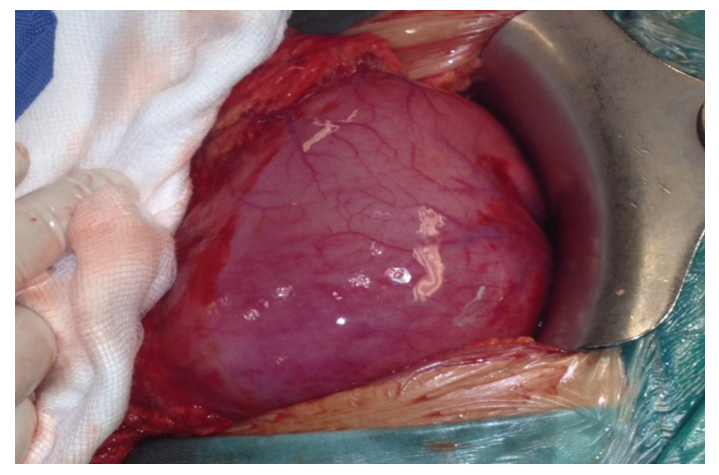

FIGURE 4: The untwisted distended stomach with ischemic mucosal lesions.

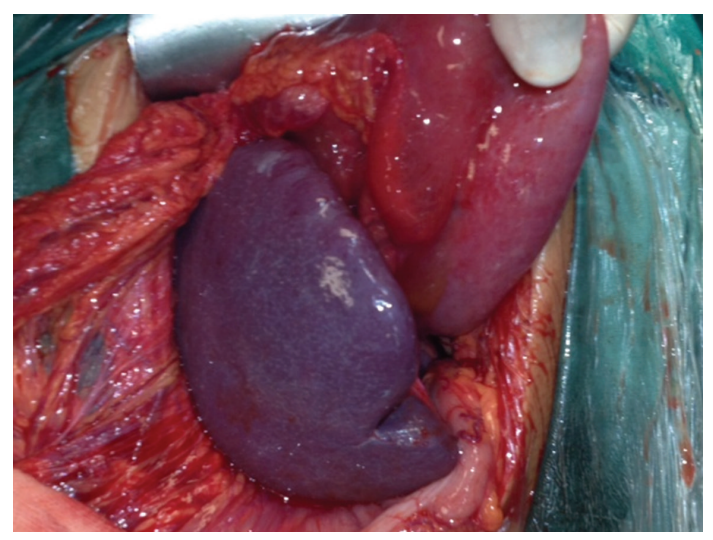

FIGURE 5: The wandering spleen.

of the stomach around another of more than 180 degrees is defined gastric volvulus [7]. This infrequent entity is in almost all the cases associated with congenital diaphragmatic hernia and eventration of the diaphragm. Interestingly, there is also a rare association between gastric volvulus and wandering spleen. These entities share a common cause, the absence or laxity of intraperitoneal visceral ligaments. Wandering spleen is a mobile spleen attached only by its vascular pedicle. This spleen can migrate to any part of the abdomen [8]. Gastric volvulus can be acute, chronic, or acute on chronic. Acute gastric volvulus is more rare than chronic. 




Figure 6: The approximation of the gastroesophageal junction and pylorus, leading to gastric volvulus.

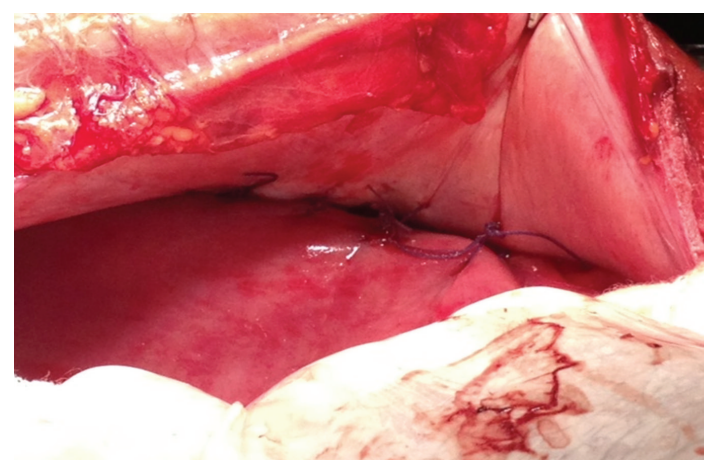

FIGURE 7: Anterior gastropexy.

There are described 3 types of gastric volvulus according to the axis of rotation: organoaxial, mesenteroaxial, and combination. Organoaxial volvulus is the most common and occurs in approximately $59 \%$ of cases. Because the duodenum and gastroesophageal (GE) junction are relatively fixed, the stomach rotates around the longitudinal axis extending from the gastroesophageal junction to pylorus. The mesenteroaxial volvulus is presented in $29 \%$ of cases. The rotation occurs around the transgastric axis (a line connecting the middle of the lesser curvature with the middle of the greater curvature). It is reported that most cases of chronic gastric volvulus are related to mesenteroaxial rotation. Additionally is described in the literature that a normal stomach cannot rotate more than $180^{\circ}$ unless the gastrosplenic or gastrocolic ligaments are divided $[9,10]$. The aetiology of gastric volvulus is thought to be secondary to laxity or lack of the gastric (gastrohepatic, gastrosplenic, gastroduodenal, and gastrophrenic) ligaments, allowing approximation of cardiac and pyloric ends when the stomach is full, leading to volvulus, as in our case (Figure 7) [11]. The clinical picture of gastric volvulus may occur as an acute abdominal emergency or as recurrent volvulus. In the literature, the famous Borchardt triad that is present typically in cases of acute gastric volvulus is described. This triad includes severe epigastric pain with distention, vomiting followed by violent, nonproductive retching, and finally difficulty or inability to pass a nasogastric tube into the stomach. Moreover, a missed diagnosis of gastric volvulus may lead to strangulation, perforation, hemorrhage, ischemia, and gastric necrosis [12]. The mortality rate of gastric volvulus is reported to be up to $42-56 \%$, secondary to gastric ischaemia, perforation, and necrosis [13]. The diagnosis of this rare clinical entity is very challenging. The gold standard method in detecting gastric volvulus is a barium swallow, which has a very high sensitivity and specificity. Additionally, highly suggestive of gastric volvulus is the difficulty during endoscopy to intubate the stomach or the pylorus. As for the treatment, gastric volvulus presenting with acyte symptoms requires immediate surgical intervention. The most approved surgical treatment consists in anterior gastropexy with open or laparoscopic technique. During this method, the greater curvature of the stomach is fixed to the anterior abdominal wall. Subtotal or total gastrectomy is proposed when the stomach appears gangrenous [14, 15].

\section{Conclusion}

Though rare, gastric volvulus must be always considered in the differential diagnosis when a patient with the Borchardt triad is admitted to the hospital. A missed or delayed diagnosis may result in unfavorable outcomes. It seems that the most important factor in diagnosing gastric volvulus is the awareness of its possibility. The diagnosis is suspected mainly by symptoms and exclusion of other pathologies. Surgical intervention is the optimal treatment. Additionally, gastric volvulus is rarely associated with wandering spleen that may lead to splenic torsion. These entities are potentially life-threatening, if not treated in time.

\section{Consent}

A written informed consent was obtained from the patient for publication of this paper and accompanying images. A copy of the written consent is available for review by the Editor-inChief of this journal.

\section{Conflict of Interests}

The authors declare that they have no conflict of interests.

\section{Authors' Contribution}

All authors were actively involved in the preoperative and postoperative care of the patient, read and approved also the final manuscript form.

\section{References}

[1] A. Berti, "Singolare attortigliamento dell' esofago col duodeno seguita da rapida morte," Gazzetta Medica Italiana, vol. 9, p. 139, 1866.

[2] S. Gourgiotis, V. Vougas, S. Germanos et al., "Acute gastric volvulus: diagnosis and management over 10 years," Digestive Surgery, vol. 23, no. 3, pp. 169-172, 2006.

[3] B. Chau and S. Dufel, "Gastric volvulus," Emergency Medicine Journal, vol. 24, no. 6, pp. 446-447, 2007. 
[4] J. M. Spector and J. Chappell, "Gastric volvulus associated with wandering spleen in a child," Journal of Pediatric Surgery, vol. 35, no. 4, pp. 641-642, 2000.

[5] M. E. Zimmermann and R. C. Cohen, "Wandering spleen presenting as an asymptomatic mass," Australian and New Zealand Journal of Surgery, vol. 70, no. 12, pp. 904-906, 2000.

[6] N. O. Machado and B. A. Rao, "Gastric volvulus with identifiable cause in adults. Presentation and management," Saudi Medical Journal, vol. 25, no. 12, pp. 2032-2034, 2004.

[7] R. Carter, L. A. Brewer, and D. B. Hinshaw, "Acute gastric volvulus: a study of 25 cases," American Journal of Surgery, vol. 140, no. 1, pp. 99-106, 1980.

[8] G. Pelizzo, M. A. Lembo, A. Franchella, A. Giombi, F. D’Agostino, and S. Sala, "Gastric volvulus associated with congenital diaphragmatic hernia, wandering spleen, and intrathoracic left kidney: CT findings," Abdominal Imaging, vol. 26, no. 3, pp. 306-308, 2001.

[9] L. W. Milne, "Gastric volvulus: two cases and a review of the literature," Journal of Emergency Medicine, vol. 12, no. 3, pp. 299306, 1994.

[10] J. A. Wasselle and J. Norman, "Acute gastric volvulus: pathogenesis, diagnosis, and treatment," American Journal of Gastroenterology, vol. 88, no. 10, pp. 1780-1784, 1993.

[11] W. J. Teague, R. Ackroyd, D. I. Watson, and P. G. Devitt, "Changing patterns in the management of gastric volvulus over 14 years," British Journal of Surgery, vol. 87, no. 3, pp. 358-361, 2000.

[12] M. Borchardt, "Zur pathologie und therapie des magen volvulus," Langenbecks Arch Klin Chir Ver Dtsch Z Chir, vol. 74, pp. 243-260, 1904.

[13] S. Farag, V. Fiallo, S. Nash, and F. Navab, "Gastric perforation in a case of gastric volvulus," American Journal of Gastroenterology, vol. 91, pp. 1863-1864, 1996.

[14] A. R. Askew, "Treatment of acute and chronic gastric volvulus," Annals of the Royal College of Surgeons of England, vol. 60, no. 4, pp. 326-328, 1978.

[15] C. Palanivelu, M. Rangarajan, A. R. Shetty, and R. Senthilkumar, "Laparoscopic suture gastropexy for gastric volvulus: a report of 14 cases," Surgical Endoscopy and Other Interventional Techniques, vol. 21, no. 6, pp. 863-866, 2007. 


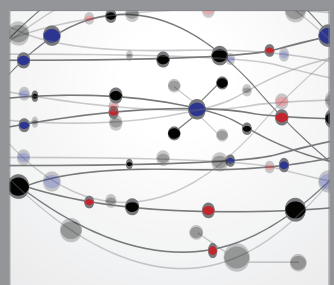

The Scientific World Journal
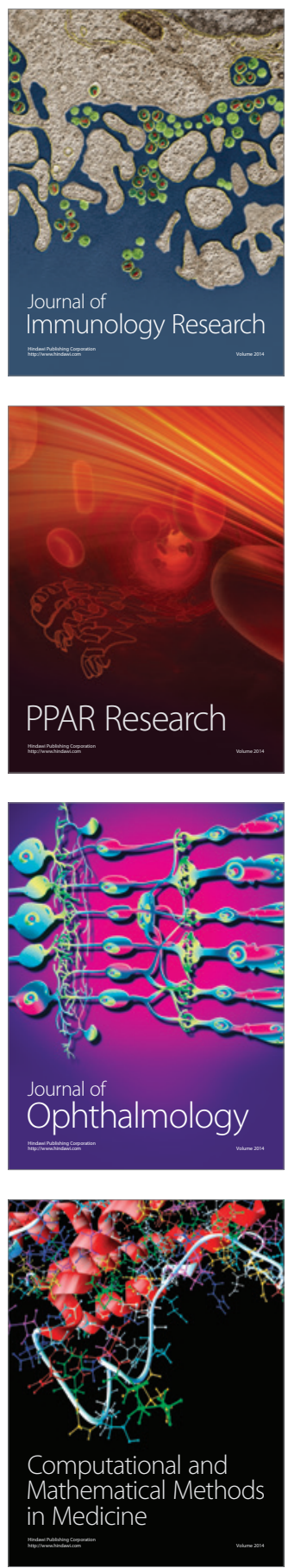

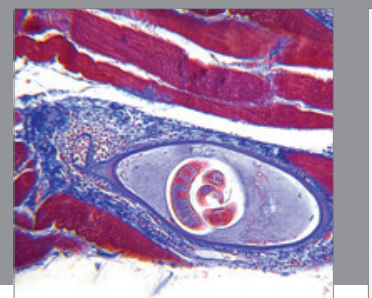

Gastroenterology

Research and Practice
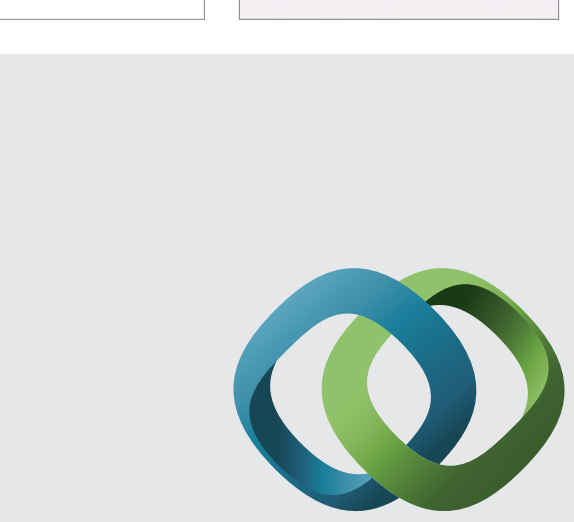

\section{Hindawi}

Submit your manuscripts at

http://www.hindawi.com
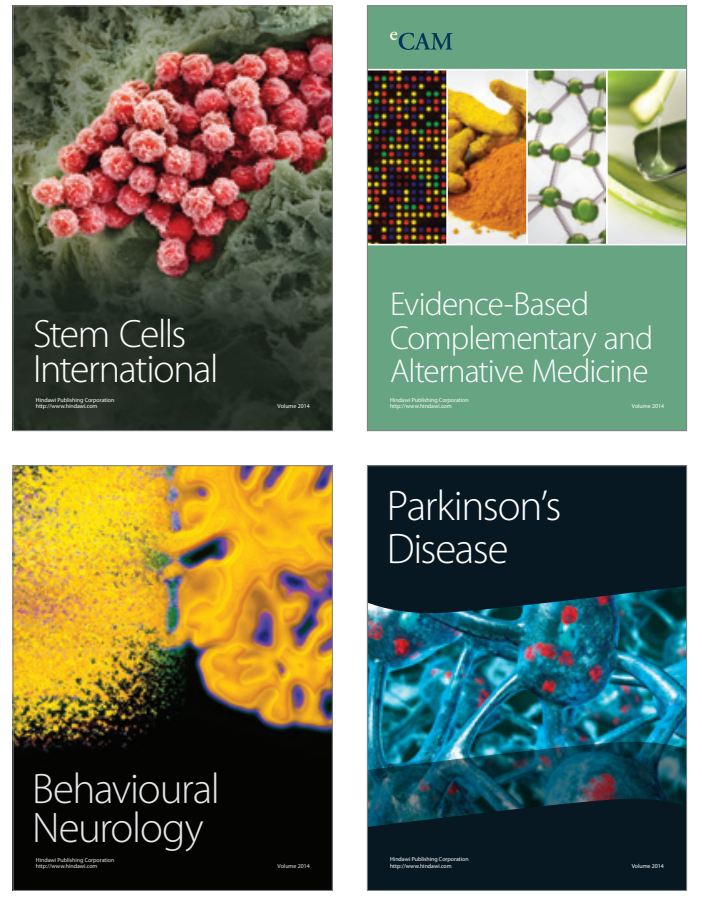


Disease Markers
